\title{
BIM Engineering Cost Budgeting Method based on Social Network Analysis Method
}

\author{
Guohui Fan*, Chen Guo, \\ Henan Polytechnic Institute, \\ Nanyang 454000 \\ China \\ *E-mail of corresponding author: beautifybird257@ 126.com
}

Received: July 2, 2021. Revised: December 19, 2021. Accepted: January 5, 2022. Published: January 7, 2022.

\begin{abstract}
Engineering cost budgeting is crucial to the healthy development of the engineering and construction industry. To solve the problem of large deviations in budget results from using traditional methods of cost budgeting based on evaluation results or drawing conversions, the BIM engineering cost budgeting method based on social network analysis method is studied in this paper. On the basis of analyzing cost budgeting requirements and determining modeling economic criteria, the social network analysis method is used to analyze the project cost risks. According to the project quantity list, professional BIM software is used to budget the project cost. Case verification proves the feasibility of this budgeting method as the budget deviation is reduced by about $\mathbf{7 8 \%}$ compared with the traditional method. The result of the paper has a more ideal practical application effect and positive significance for optimizing the traditional cost budgeting methods.
\end{abstract}

Keywords-Social network analysis; BIM software; Construction engineering; Cost budget; Project cost risk; Economic criteria for modeling.

\section{INTRODUCTION}

The budget management of China's construction projects, although after decades of development, the budget management methods have been improved in the past practice. However, the development level of the whole engineering cost industry still has a gap with the current level of economic and social development, and there are differences with the development of the industry, one of the important reasons is that the degree of informationization and refinement of cost management is not enough and not high enough. This situation restricts the improvement of the accuracy and efficiency of China's engineering cost, and to a certain extent affects the healthy development of China's construction engineering industry. If we can realize the process of implementing cost control in the whole process of engineering budget management from estimation to budget and even final account, realize accurate engineering budget, realize refined engineering project budget management, then we can realize effective cost control, which can be more ideal to cut unnecessary expenses in the project and include the control of risks. However, the actual situation is that: the budget estimate, budget, final account overrun, suppliers fly single, material consumption over the fixed consumption and other phenomena are very common, seriously affecting the budget accuracy of engineering costs. For the above realistic problems, there are also some traditional engineering cost control methods applied to the project: applying the principle of earned value, comparing the actual cost, schedule and the planned one; adopting standardized design and implementing limit design to make a dynamic control of the project cost, etc. [1]. However, under the traditional management system and working methods, some of these cost control methods are constantly highlighted in the implementation, and it is difficult to effectively obtain the planned cost and schedule information of each node for real-time control of project cost based on the earned value principle; for the limit design, it is difficult to reasonably decompose the design index, which leads to such an active cost control program as the limit design becoming a passive implementation. The program becomes passive implementation, etc. Therefore, some such methods cannot effectively control the three super phenomena in the process of actual construction budget in actual projects.

In order to fundamentally solve the problem of budget cost information disconnection of construction projects, improve the level of engineering cost management and engineering cost efficiency, effectively realize the accurate and intelligent budget of engineering cost, realize the whole process of engineering cost control, realize the refined engineering budget management, and better analyze the economic data of engineering budget, it is necessary to find an effective plan to put some existing methods, software, tools, etc. on a unified platform. BIM, as an emerging technology, provides such an integrated platform for construction information and has shown its strong potential and all-round advantages in various practical applications. Compared with other engineering modeling 
methods, the level of accuracy of modeling construction projects using BIM is higher. If the calculation process of engineering quantity is too tedious, there is a greater chance for engineers to make mistakes, which in turn will cause the accuracy level of engineering quantity to decrease. With the continuous development and application of BIM, BIM engineering has become the mainstream form of current construction engineering design [2].

Many scholars and experts at home and abroad have made a lot of research results in this research of BIM engineering cost budget. In the literature [3], the BIM engineering cost budgeting method by improving cost management is mentioned, and the accuracy of its cost budgeting depends on the accuracy of assessing the cost level of the project. The evaluation of the cost level of the project will be affected by subjective factors, and the cost control requirements for each aspect of the BIM project are high and not applicable to the more complex project budget. The budget method mentioned in the literature [4] is to use 3D software to achieve the effect of 3D solid roaming of the building model, so as to directly extract the cost and design information of the project to complete the project cost budget. However, the 3D software used in this budgeting method is usually modeled by identifying drawings, and the recognition rate of drawings is not high, resulting in low modeling accuracy and leading to large deviations in cost budgeting results.

To solve the above problems, regarding the above analysis, this paper will study the BIM engineering cost budgeting method based on social network analysis method. Social network analysis is a theory that can be used to study the interrelationships between actors or organizations and the structure and properties of social networks composed of these relationships by quantifying the relationships in social networks [5]. The idea of social network analysis is different from the traditional atomistic analysis theory, which takes a single individual as the object of study, and is characterized by its focus on the interactions and connections between individuals or organizations and its emphasis on studying the social behavior of individuals with a structural and holistic perspective. A social network is a structure of relationships generated by the interconnectedness of individuals or organizations in society. For actors in a social network, their decisions and actions are interdependent rather than independent. Combining social network analysis with BIM engineering cost budget can improve the accuracy of engineering cost budget by starting from the project itself. So, the result of the paper has a more ideal practical application effect and positive significance for optimizing the traditional cost budgeting methods.

II. RESEARCH ON BIM ENGINEERING COST BUDGETING Method BASEd on SOCIAL NETWORK ANALYSIS METHOD

\section{A. BIM project costing model establishment}

(1) BIM engineering modeling economic standard setting The purpose, direction and focus of the practical application of BIM vary from country to country according to their needs, but what is very consistent is that each country establishes BIM usage standards suitable for their engineering construction conditions to pave the way for the use, research and development of BIM in their countries. The use of BIM technology and BIM modeling research in China is mainly to combine BIM technology with engineering budget. Therefore, before cost budgeting of BIM projects, a BIM modeling standard suitable for domestic budget management in China BIM modeling economic data standard - should be established. Hereinafter, this paper innovatively defines the BIM modeling economic data standard as follows: for the basic engineering budget measurement and valuation in China, propose some BIM modeling requirements that are specifically applicable to China, conform to the modeling design specification, higher than the general modeling standard, and ensure that the completed model can be directly used for engineering budget, accounting and other budget management system, and further form the modeling standard to realize the BIM technology effectively used for engineering budget management in China [6].

BIM modeling economic data standards will be more stringent than the general BIM modeling standards, because it is proposed not only to establish a perfect, standardized and standard BIM model, but to achieve the cost budget in this model to the maximum extent by the most rapid and effective way, so this will undoubtedly put forward higher requirements for BIM modeling, what are the higher requirements to form BIM modeling economic Data standards this is what is studied later in this paper. To use BIM model for engineering budget, it is necessary to make this technology introduced from abroad fit with the current measurement and pricing of engineering budget in China, and to achieve this fit, it will put requirements on the implementation of this technology [7]. The actual use of BIM for engineering budgeting will encounter various modeling problems in both measurement and pricing, and the solution to these problems boils down to the need to put forward requirements for BIM modeling. If the modeling standards of BIM can be further proposed based on these requirements, this can provide standard support and specification requirements for the widespread use of BIM technology in China.

(2) BIM engineering cost budget requirement analysis

For the cost budget of construction engineering, BIM technology, data mining technology and big data technology are widely used at present. However, due to the high requirements of information integration in construction engineering, the application of data mining technology and big data technology is not ideal. Building Information Modeling is a new tool for architecture, engineering, and civil engineering. The core of BIM is to provide a complete and consistent construction information base for this model by establishing a virtual three-dimensional model of construction project and using digital technology. The information base contains not only the geometric information, professional attribute and state information of building components, but also the state information of non-component objects (such as space, motion 
behavior). With the help of the 3D model which contains the construction information, the integration level of the construction information is greatly improved.

The methods of BIM engineering cost budget are mainly divided into two categories, namely direct methods and indirect methods. The former refers to obtaining the necessary data already available to calculate the cost directly, while the latter refers to obtaining a small amount of necessary data to make a rough estimate of the cost through mathematical statistics and other methods. According to the above description, the application process of the direct method is simpler than that of the indirect method, and the limitations are smaller. Therefore, this study mainly considers the direct method. The direct method of preparing construction budget is widely used in the actual process in China, namely, the fixed-price method and the bill-of-contract method. The former is mainly used to determine the unit price of sub-projects according to local quotas and summarize the direct cost of the project, and then determine the cost of the project, which is characterized by the combination of quantity and price; the latter is used to give the list of quantities according to the list specification, and the tenderer gives the comprehensive unit price according to the list of quantities in combination with the enterprise quotas, and finally summarizes the cost of the project, which is characterized by the separation of quantity and price. The direct method of preparing construction budget is mainly the physical method, in which the amount of labor, materials and machinery is determined and summarized based on the national unified construction quota or enterprise quota in the application, and the total cost is calculated by combining with the actual price [8-9]. No matter which method is used, the basic principle is the same, that is, the project is divided into cost items according to certain rules, and its volume is calculated, then the consumption or cost of cost items is derived by combining the resource consumption standards (quotas), and finally the total cost is obtained by aggregating according to certain methods. The differences between different methods are mainly reflected in the different fineness of cost item division, the different composition of cost items, the different aggregation methods of total project cost, the different price taking methods and the different norms based on. Based on this principle, this paper will analyze the BIM engineering cost budget requirements according to the framework shown in Figure 1 [10].

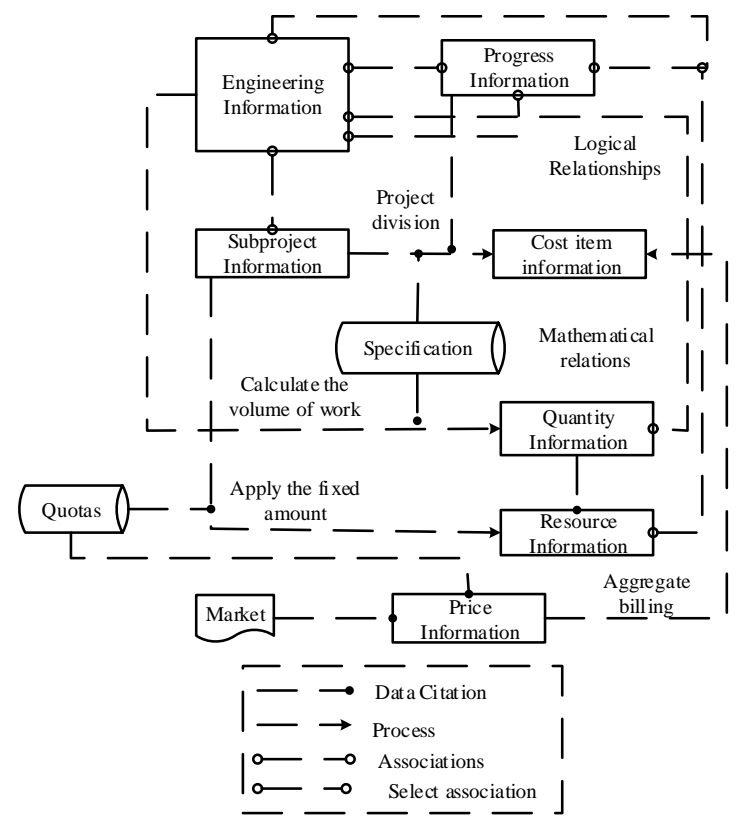

Fig. 1. BIM engineering cost budget requirement analysis framework

As can be seen from Figure 1, a total of seven areas of information are required for construction project cost budgeting. information on BIM engineering products is essential as information for the design phase. In addition, the information of subdivision of products is also needed to determine the corresponding cost item information and to carry out the calculation of engineering quantity information and resource information. The information directly required for cost estimation includes quantity information, resource information, and price information. The quantity information is based on the construction product information and subdivision information and is calculated according to the specifications. Resource information, i.e., information on the quantity of materials consumed, labor and machinery required for the construction project, is generally obtained by applying the quotas and using the quantity information [11]. Price information is mainly derived from the market, and some local quotas already contain price data, which are used in the pricing process. Progress information is generally applied to the construction budget, and the division of cost items can be made based on progress tasks, which makes the cost item expression with time dimension, and the expression of cost is achieved by establishing the relationship between cost items and progress, progress and products.

\section{B. Social network-based BIM engineering cost risk analysis}

Social networks view BIM engineering as a system environment consisting of various relationships. The purpose of network analysis is to examine how the structure of relationships affects behavior. Social network analysis is used to analyze the structure and patterns of the various relationships included in the BIM engineering cost budgeting process in an attempt to determine the reasons for the relationship structure and its impact.

Using social network analysis to examine the structure of 
relationships between risk factors related to stakeholders requires the creation of a model for social network analysis. And the basic steps of social network analysis include: (1) identifying the boundaries of the network, (2) determining the links between risk factors, (3) visualizing the network, (4) analyzing the network data, and (5) presenting the results of the analysis. In this paper, we will analyze the risks in BIM engineering cost budgeting process using social network analysis method according to the above process.

(1) Stakeholder and related risk factor identification

For different BIM projects, there are many factors that affect the project cost budget, and identifying the stakeholders and related risks that have a significant impact on the BIM project cost is the basis for the BIM project cost budget. Relevant research shows that there are three types of people in the project environment, and according to social network theory, this paper is expressed through three tandem circles. In the first circle are people we know well; in the second circle are people we know but are not familiar with, but the people in the first circle have a more familiar relationship with the people in the second circle; in the third circle are people we do not know, but the people in the first and second circles know each other. Based on this, stakeholders can be identified in three steps [12-13].

First, the project manager recommends stakeholder groups; then, internal stakeholders recommend external stakeholders, where external stakeholders are those outside the construction project that can influence or be influenced by the project; and finally, external stakeholders recommend others (external stakeholders) that are not mentioned by internal stakeholders and can influence or be influenced by the construction project. Rolling sampling yields a complete list of stakeholders. Questionnaires with internal and external stakeholders can be used to identify cost-related risks and stakeholders associated with this risk.

\section{(2) Clustering of risk factors}

The systematic clustering process of risk factors related to BIM engineering is as follows, using Euclidean distance between sample points and the shortest distance method between classes. In order to eliminate the influence of different magnitude indicators on the distance, the common processing method is to standardize the sample data, that is, each sample value of each indicator is subtracted from its mean value and then divided by its standard deviation, so that all sample individual indicators are transformed to a mean value of 0 and a standard deviation of 1 . The formula for calculating the Euclidean distance is shown below [14].

$d_{i j}=\sqrt{\sum_{k=1}^{p}\left(x_{i k}-x_{j k}\right)^{2}}$

In equation (1), $x_{i k}$ and $x_{j k}$ are different data in the sample data set, respectively. $p$ is the total amount of data in the sample data set. The shortest Euclidean distance is the distance between the closest points in two classes is noted as the distance between two classes and is calculated as follows [15].

$$
D(i, j)=\min \left\{d_{i j} \mid i, j \in G_{j}\right\}
$$

In the above equation, $D(i, j)$ is the shortest Euclidean distance. The clustering steps of BIM engineering cost budget risk factors are as follows.

(1) The eight samples are divided into eight categories, numbered F1 to F8, where F1 is 'construction unit proposes engineering changes', F2 is 'construction funds are not available on time', F3 is 'construction schedule requirements are too tight difficult to meet', F4 is 'insufficient experience in project management', F5 is 'inefficient communication between project parties', F6 is 'unclear contract terms', F7 for 'unreasonable interference of construction unit supervisors/leaders in construction', and F8 for 'inaccurate original information provided by the construction unit'. Standardizing these eight samples [16-17].

(2) Calculate the inter-class distance matrix D using the standardized data according to the Euclidean distance formula.

(3) Merge the risk samples that satisfy the minimum interclass distance classification into new classes based on the calculation results in (2).

(4) Repeat the above steps until the inter-class distance is greater than 1 and stop. Considering the reasonable number of risk factors and the clustering criteria among unified stakeholders, no more clustering is done when the distance between factors is greater than 1. Output the final BIM engineering cost budget risk classification.

(3) Risk correlation analysis

Using social network analysis to determine the interconnection between risk factors, according to the social network principle the connection between risk factors is represented by defining the influence link between two nodes in the risk network. In the BIM engineering cost budget risk social network, the actors (nodes) are the risk factors identified in the first step. There are three basic types of relationships in each pair of risk factors in the organizational structure (1) independent: risk factors are not connected to each other; (2) dependent: two risk factors have a direct influence on each other; (3) interdependent: risk factors interact with each other directly or through a larger cycle. In this paper, the 0-1 matrix will be used to represent the interactions between risk factors, where 0 indicates that the active risk factor has no direct/indirect influence on the passive risk factor; 1 indicates that the active risk factor has direct or indirect influence on the passive risk factor.

Once the nodes and linkages in the cost budget risk social network are identified, the network of risk factors affecting the cost of the construction project is basically formed. A graph $G$ consists of two sets of information. The set of nodes is $N=\left\{n_{1}, n_{2}, \cdots, n_{N}\right\}$ and the set of edges between pairs of nodes is $L=\left\{l_{1}, l_{2}, \cdots, l_{L}\right\}$. Therefore, a graph is represented by the set of nodes $\mathrm{N}$ and the set of edges $\mathrm{L}$, denoted as $G(N, L)$. The nodes of different shapes represent different stakeholder risks, and the interconnections between risk factors are represented by arrow lines, where the arrows point to the affected or passive parties. A directed line segment from $S^{*} R_{i}$ to $S^{\#} R_{j}$ indicates the presence of influence between two nodes/risk factors [18]. 
Assuming the existence of three nodes $j, k$, and $l$, the intermediary centrality of node $k$ can be defined as the ratio of the geodesic lines passing through node $k$ and connecting the two nodes $j$ and $l$ to the total number of geodesic lines between the two nodes $j$ and $l$. The number of geodesic lines between node $j$ and node $l$ is denoted by $g_{j l}$ and the ability of node $k$ to control the interaction between these two nodes is denoted by $Q_{j l}(k)$, the probability that node $k$ is on the shortest path between node $j$ and node $l$. The number of geodesic lines that exist between node $j$ and node $l$ passing through node $k$ is denoted by $g_{j l}(k)$. Then, the intermediary centrality of node $k$ is expressed by the formula [19].

$Q_{j l}(k)=\frac{g_{j l}(k)}{g_{j l}}$

If the absolute intermediate centrality $C_{a b k}$ of node $k$ is calculated, it is sufficient to add together the intermediates corresponding to all pairs of nodes in the cost-budget risk graph, and the process can be expressed by the following equation.

$C_{A B k}=\sum_{j}^{n} \sum_{l}^{n} Q_{j l}(k), j \neq k \neq l, j<l$

If the relative intermediary centrality is calculated, it is sufficient to divide the absolute intermediary centrality by the maximum possible absolute intermediary centrality, which is calculated as follows.

$C_{R B k}=\frac{C_{A B k}}{n^{2}-3 n+2}$

Proximity centrality measures the ability of a node to reach all other nodes with the shortest path and is calculated as shown below:

$$
C_{c}\left(n_{i}\right)=\left[\sum_{j=1}^{g} d\left(n_{i}, n_{j}\right)\right]^{-1}
$$

In equation (6), $d\left(n_{i}, n_{j}\right)$ represents the distance between $n_{i}$ and $n_{j}$. Unlike point centrality and intermediary centrality, the smaller the proximity centrality value of a node, the more the node is at the core of the network, thus the less it is controlled by other nodes and the less it is influenced by other factors in the risk factor structure network; if the proximity centrality value of a node is larger, the more the node is at the edge of the network and the more it is controlled by other nodes and the more it is influenced by other factors in the risk factor structure network. The greater the control of other nodes, the greater the influence of other factors in the risk factor structure network. After analyzing the problems in the cost budget of BIM project by using social network analysis method, the cost budget of BIM project is completed according to the above analysis results.

\section{Complete BIM project cost budget}

The steps of basic BIM project costing are as follows: firstly, you need to design and build the BIM model of architecture, structure, electromechanics and so on of the project directly with BIM software design under the premise of understanding the basic situation of the project, and the model must be set based on the economic data standard of BIM engineering modeling in part IA(1); then, the WBS decomposition of the model is carried out to produce the constructed sub-component project list, and corresponding to the standard list to find out the list code corresponding to each sub-component project to establish the BIM-based engineering budget coding system; then, the information in the BIM system model is exported to excel to get the BIM-based project quantity table, this table is the quantity of works counted in the mode of list table; finally, the quantity table is imported into the pricing software to get the budget cost of the project [20-21].

According to the above ideas, this project firstly based on the economic data standard requirements to model BIM high requirements, and set the decoration layer in the model according to the design requirements. Then add structural members such as beams, slabs and columns, and input parameters such as dimensional loads; check the floor openings and set up the construction requirements; add the foundation model and input parameters such as foundation loads and foundation bearing capacity into the model; analyze and calculate the reinforcement through the software and generate the plain construction drawings.

Then, on top of the built model, the professional equipment and pipelines are arranged in accordance with certain requirements or certain functions stated in the design documents. Finally, collision check and design optimization are carried out. Using collision check software, the 3D model of building, structure and equipment is checked for pipeline collision, and the collision check report is generated to optimize the design and complete the BIM modeling of the project.

Based on the BIM modeling economic data standard, the BIM engineering model built according to the standard setting requirements can be exported to various solid components, decoration layer details. For the decomposition of construction projects can be through the WBS method, here for the decomposition of the BIM model can also be used WBS decomposition, the BIM model according to certain standards for continuous subdivision layer by layer, until the decomposition to the most basic smallest unit component items, and this smallest unit project is based on the ability to quickly and easily calculate the corresponding amount of work and can The minimum unit project is based on the ability to quickly and easily calculate the amount of work and effectively apply the corresponding unit price for pricing as the basis to set. In this way, the cost of each unit item can be aggregated to obtain the cost of the physical project of the BIM model.

The flow chart of WBS decomposition and cost summary of specific BIM model is shown in Figure 2. 


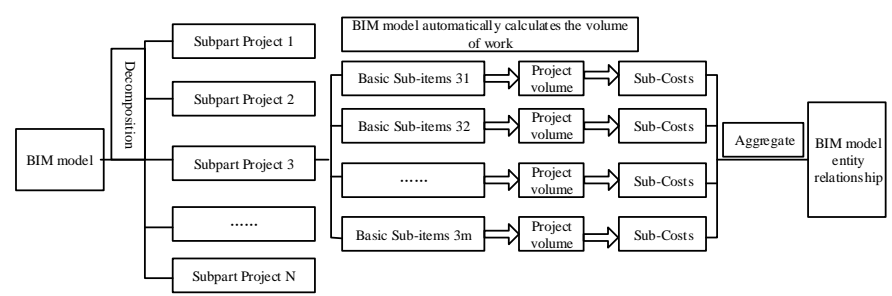

Fig. 2. BIM model budget costing flow chart

In actual operation, the model can be decomposed according to the above WBS decomposition process firstly according to the divisional works in the list, and then further decompose the corresponding model parts in each divisional works according to the items listed in the itemized works in the list, so as to determine to which itemized works and which divisional works in the list each component of the model is categorized. According to the decomposition result, the list-based coding is set for each component in the BIM model, and the quantities of the components in the model are summarized according to each code. After that, the BIM database of "3D entity + time + WBS" is created with WBS as the main line, and cost budgeting is carried out according to the following contents.

Create BIM-based actual cost database. Based on the 3D solid model of BIM project, the model is decomposed into WBS, and Nevisworks software is used to add time and process information into the model components to create a 5D (3D solid + time + process) related database of cost, which contains various plans and targets, including planned processes, planned project schedule, etc. In this 5D database, the actual cost data of the process will be inputted in time, and the system will automatically and quickly carry out the cost classification and statistics of the project. The WBS decomposition of the model will be used to input the unit cost of men, materials and machines as the main data into the 5D database of BIM. Through the above steps, the calculated result is the final budget result of the BIM project cost. At this point, the research on BIM engineering cost budgeting method based on social network analysis method is completed.

\section{CASE VAlidation STUdy}

BIM technology is gradually applied in different engineering constructions because of its convenience and modifiability. In order to more accurately account for the cost of construction projects, the BIM engineering cost budgeting method based on social network analysis method is proposed above. In this section, the feasibility of this cost budgeting method will be verified by selecting actual engineering cases.

\section{A. Brief description of experimental contents and steps}

This instance validation will be in the form of comparison in order to make the final evaluation of the feasibility of cost budgeting methods objective and scientific. The engineering cost budgeting methods mentioned in literature [3] and literature [4] are selected as comparison group 1 and comparison group 2, respectively, and the BIM engineering cost budgeting method studied above is the experimental group. The comparison index of this experiment is the cost budget of three engineering cost budgeting methods for the same construction project respectively, and the calculation accuracy of three cost budgeting methods are compared according to the real cost of each stage of actual engineering construction.

During the instance verification, the relevant experimental variables are controlled uniquely. According to the specific processing calculation process of the 3 engineering budgeting methods respectively, the cost budget of different construction aspects of the project is carried out according to the specific parameters of the experimentally selected construction project. The cost budget values calculated by the 3 methods are compared with the costs in the actual construction process of the project, and the accuracy of the 3 cost budgeting methods is compared by comparing the difference between the budgeted and actual costs of the project. The closer the calculated cost budget value is to the actual cost in the construction process, the better the application effect of this method will be.

\section{B. Project profile}

The experimental project is an office complex, i.e. office building project, developed by a real estate company and designed by an architectural design company in the city. It consists of four high-rise office buildings of more than 20 floors high respectively, and this case study is mainly based on one of the A1 office buildings.A1 office building has an above-ground construction area of 206502 square meters and an underground construction area of 86512.6 square meters, with a total construction area of 293014.6 square meters and a building height of 98.55 meters, with an engineering design elevation of ground level equivalent to the absolute Elevation 1290m, 23 floors above ground and 2 floors below ground. The building category is a class of high-rise, the structure is designed as shear wall structure, the seismic intensity is 6 degrees, the fire resistance level is one, and the service life is 50 years.

\section{Case verification results and analysis}

The cost budgets of the three engineering cost budgeting methods for each construction operation of the same project and the differences between the budgeted and actual cost budgets are shown in Table 1 below. A positive budget difference in the table means that the budget value of the budget method is greater than the actual cost value, and a negative difference means that the budget value of the budget method is less than the actual cost value. The data in Table 1 were analyzed to draw the final conclusions. 
Table 1 Comparison of the data from the instance validation analysis

\begin{tabular}{|c|c|c|c|c|c|c|c|}
\hline \multirow{2}{*}{$\begin{array}{l}\text { Construction } \\
\text { link number }\end{array}$} & \multirow{2}{*}{$\begin{array}{c}\text { Actual } \\
\text { construction } \\
\text { cost/ million } \\
\text { yuan }\end{array}$} & \multicolumn{2}{|c|}{ Comparison group 1} & \multicolumn{2}{|c|}{ Comparison group 2} & \multicolumn{2}{|c|}{ Experimental group } \\
\hline & & $\begin{array}{l}\text { Budget value/ } \\
\text { million yuan }\end{array}$ & $\begin{array}{l}\text { Difference/ } \\
\text { million }\end{array}$ & $\begin{array}{l}\text { Budget value / } \\
\text { million yuan }\end{array}$ & $\begin{array}{l}\text { Difference/ } \\
\text { million }\end{array}$ & $\begin{array}{l}\text { Budget } \\
\text { value/ } \\
\text { million } \\
\text { yuan }\end{array}$ & $\begin{array}{l}\text { Difference } \\
\text { / million }\end{array}$ \\
\hline 1 & 203236.01 & 245869.25 & 42633.24 & 227869.37 & 24633.36 & 208974.24 & 5738.23 \\
\hline 2 & 18808279.15 & 18617243.86 & -191035.29 & 19024135.62 & 215856.47 & 18813425.70 & 5146.55 \\
\hline 3 & 1471779.23 & 1752764.35 & 280985.12 & 1531426.17 & 59646.94 & 1480512.01 & 8732.78 \\
\hline 4 & 477183.68 & 464007.72 & -13175.96 & 510236.43 & 33052.75 & 477572.40 & 388.72 \\
\hline 5 & 311233.05 & 334243.15 & 23010.1 & 323986.89 & 12753.84 & 314072.11 & 2839.06 \\
\hline 6 & 4200695.72 & 4358695.72 & 158000 & 436586.63 & $\begin{array}{c}-3764109.0 \\
9\end{array}$ & 4280479.87 & 79784.15 \\
\hline 7 & 3295616.74 & 3510271.36 & 214654.62 & 3452423.64 & 156806.9 & 3312456.76 & 16840.02 \\
\hline 8 & 521292.13 & 529975.95 & 8683.82 & 525487.85 & 4195.72 & 521781.15 & 489.02 \\
\hline 9 & 872657.28 & 897864.38 & 25207.1 & 885769.52 & 13112.24 & 872778.74 & 121.46 \\
\hline 10 & 692660.97 & 712543.81 & 19882.84 & 699853.78 & 7192.81 & 694215.63 & 1554.66 \\
\hline 11 & 119375.53 & 127687.20 & 8311.67 & 124334.67 & 4959.14 & 120375.62 & 1000.09 \\
\hline
\end{tabular}

\section{DISCUSSION}

Through the above experimental results, it can be seen that among the various aspects of the construction project, the deviation between the budget result and the actual value of the comparison group method 1 is the largest among the three methods, the deviation value between the budget result and the actual value of the comparison group method 2 is in the middle size, and the deviation of the budget result of the experimental group method is the smallest. Among the three budgeting methods, the budgeted cost of the comparison group method 1 and the comparison group method 2 has a low budgeted value, while the budgeted cost of the experimental group method only has a large deviation, and the deviation value is much smaller than the other two methods. And in the actual engineering construction, the case of large cost budget is better than the case of small cost budget. Calculating the deviation of the total cost of the whole construction project budget, the budget accuracy of the experimental group method is improved by about $78.7 \%$ compared with the other two groups of methods.

Based on the above research and analysis content, the proposed BIM project cost budgeting method based on social network analysis method is more accurate, and the cost budgeting result obtained is consistent with the actual required cost, which proves that the proposed method has a more ideal practical application effect.

\section{V.CONCLUSION}

In the construction of engineering projects, especially in the construction of transmission and transformation projects, the management and control of engineering cost has a great impact on the progress and cost. For a long time, our country has adopted the budgetary estimate system based on quota, which is not conducive to give full play to the role of market regulation to a certain extent. The traditional substation project cost budget applies the unified quota, then determines the substation project budgetary estimate, lacks the effective management method and the incentive measure to the project construction unit and the cost management control unit, cannot display the market guidance fully the function.

Aiming at the problems of traditional BIM engineering cost budgeting methods, this paper proposes a BIM engineering cost budgeting method based on social network analysis method. By selecting specific practical cases, the feasibility of the cost budgeting method proposed in this paper is verified. The application of social network analysis method in project cost risk assessment is analyzed based on cost budgeting requirements and determining modeling economic criteria. Combined with BIM software to achieve the project cost budget. Experimental results show that the proposed method has a small budget error and is feasible.

But this paper also has some place which needs to improve and the consummation. In this paper, there are many artificial interventions in analyzing the cost budget demand and determining the economic criteria of modeling, which can not achieve real intelligence.

\section{ACKNOWLEDGMENT}

This research is supported by Research and Practice of Higher Education Reform Research and Practice Project of Henan Province in 2019, Research and Practice of Technical and Skilled Personnel Training Mode Reform Based on "1+X" BIM Certificate System (No. 2019SJGLX698). 


\section{References}

[1] S. Chatterje, B. Chaudhuri, C. Bhar. "Optimal release time determination in intuitionistic fuzzy environment involving randomized cost budget for SDE-based software reliability growth model." Arabian Journal for Science and Engineering, 2020, vol. 45, n. 9, pp. 2721-2741.

[2] M. Van der Spek, S. Roussanaly, E. S. Rubin. "Best practices and recent advances in CCS cost engineering and economic analysis." International Journal of Greenhouse Gas Control, 2019, vol. 83, pp. 91-104

[3] M. Dadpour, E. Shakeri, A. Nazari. "Analysis of stakeholder concerns at different times of construction projects using social network analysis (SNA)." International Journal of Civil Engineering, 2019, vol. 17, n. 11 , pp. 1715-1727.

[4] W. S. Jäger, T. Nagler, C. Czado, et al. "A statistical simulation method for joint time series of non-stationary hourly wave parameters." Coastal Engineering, 2018, vol. 146, pp. 14-31.

[5] V. Toğan, M. A. Eirgash. "Time-cost trade-off optimization of construction projects using teaching learning based optimization." KSCE Journal of Civil Engineering, 2019, vol. 23, n. 1, pp. 10-20.

[6] P. Cicconi, Castorani V, Germani M, et al. A multi-objective sequential method for manufacturing cost and structural optimization of modular steel towers. Engineering with Computers, 2020, vol. 36, n. 2, pp. 475-497.

[7] D. M. Azevedo, A. Marotti, A. Cardoso, et al. "Development of BIM (building information modeling) concept applied to projects of substations integrated with the geographic intelligence system (GIS)." WSEAS Transactions on Power Systems, 2021, vol. 16, Art. \#1, pp. $1-7$.

[8] J. A. Salinas-Martínez, R. R. Posadas-Domínguez, L. D. Morales-Díaz, et al. "Cost analysis and economic optimization of small-scale dairy production systems in Mexico.” Livestock Science, 2020, vol. 237, pp. 104028.

[9] M. Delavar, G. T. Bitsuamlak, J. K. Dickinson, et al. "Automated BIM-based process for wind engineering design collaboration.” Building Simulation, 2020, vol. 13, n. 2, pp. 457-474.

[10] B. Das, A. Singh, S. K. Paul, et al. "Correlation between fatigue response of preformed bend DP600 steel specimen and wheel disc." Fatigue \& Fracture of Engineering Materials \& Structures, 2020, vol. 43, n. 12, pp. 2842-2853.

[11]R. Dhurandhar, J. P. Sarkar, B. Das. "Elucidation of hydrodynamics and heat transfer characteristic of converging and equivalent uniform riser for dilute phase gas-solid flow." Chemical Engineering Research and Design, 2019, vol. 151, pp. 120-130.

[12] R. Vieira, P. Carreira, P. Domingues, et al. "Supporting building automation systems in BIM/IFC: reviewing the existing information gap." Engineering Construction and Architectural Management, 2020, vol. 27, n. 6, pp. 1357-1375.
[13] B. Youbi, Y. Lghazi, M. Ait Himi, et al. "Nucleation and growth mechanism of tellurium electrodeposited on tin-doped indium oxide substrate." Journal of Applied Electrochemistry, 2020, vol. 50, n. 2, pp. 159-168.

[14]R. X. Ren, M. Tang, H. C. Liao. "Managing minority opinions in micro-grid planning by a social network analysis-based large scale group decision making method with hesitant fuzzy linguistic information." Knowledge-Based Systems, 2020, vol. 189, pp. 105060.

[15] G. Acampa, M. Grasso, G. Marino, et al. "Tourist flow management: social impact evaluation through social network analysis." Sustainability, 2020, vol. 12, n. 2, pp. $1-1$.

[16] J. R. Enos, R. R. Nilchiani. "Understanding the importance of expanding the definition of interoperability through social network analysis." Systems Engineering, 2020, vol. 23, n. 2, pp. 139-153.

[17] S. Selva Kumar, N. Kayavizhy. "Novel approach for optimizing information propagation in dynamic social network." WSEAS Transactions on Computers, 2020, vol 19, Art. \#1, pp. 1-9.

[18]G. F. Khan, M. Sarstedt, W. L. Shiau, et al. "Methodological research on partial least squares structural equation modeling (PLS-SEM).” Internet Research, 2019, vol. 29, n. 3, pp. 407-429.

[19] J. Wongpen, J. Wetweerapong, P. Puphasuk. "Finding a maximum clique in social networks using a modified differential evolution algorithm." WSEAS Transactions on Systems and Control, 2019, vol 14, Art. \#42, pp. 333-342.

[20] K. Nagarajan, M. Muniyandi, B. Palani, et al. "Social network analysis methods for exploring SARS-CoV-2 contact tracing data." BMC medical research methodology, 2020, vol. 20, n. 1, pp. 233.

[21] W. Gao, R. N. Youngworth, J Sasian. "Method to evaluate surface figure error budget for optical systems." Optical Engineering, 2018, vol. 57, n. 10, pp. 105108.1-105108.7.

\section{Creative Commons Attribution License $\mathbf{4 . 0}$ (Attribution 4.0 International, CC BY 4.0)}

This article is published under the terms of the Creative Commons Attribution License 4.0

https://creativecommons.org/licenses/by/4.0/deed.en_US 